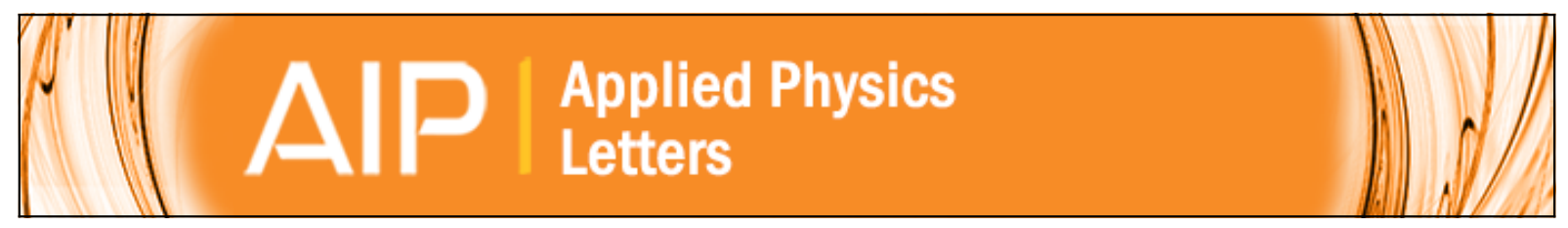

\title{
Pressure-dependent Schottky barrier at the metal-nanotube contact
}

Noejung Park, Donghoon Kang, Suklyun Hong, and Seungwu Han

Citation: Applied Physics Letters 87, 013112 (2005); doi: 10.1063/1.1990251

View online: http://dx.doi.org/10.1063/1.1990251

View Table of Contents: http://scitation.aip.org/content/aip/journal/apl/87/1?ver=pdfcov

Published by the AIP Publishing

Articles you may be interested in

Impacts of image force on the Schottky barrier height at metal-carbon nanotube contacts

Appl. Phys. Lett. 100, 173104 (2012); 10.1063/1.4705300

Schottky barrier height reduction for metal/n-GaSb contact by inserting TiO 2 interfacial layer with low tunneling resistance

Appl. Phys. Lett. 98, 172106 (2011); 10.1063/1.3584862

Temperature dependent Schottky barrier height and Fermi level pinning on Au/HBC/GaAs diodes

Appl. Phys. Lett. 92, 153309 (2008); 10.1063/1.2912062

Schottky diodes from asymmetric metal-nanotube contacts

Appl. Phys. Lett. 88, 133501 (2006); 10.1063/1.2190707

Electronic structure of the contact between carbon nanotube and metal electrodes

Appl. Phys. Lett. 83, 3180 (2003); 10.1063/1.1616662

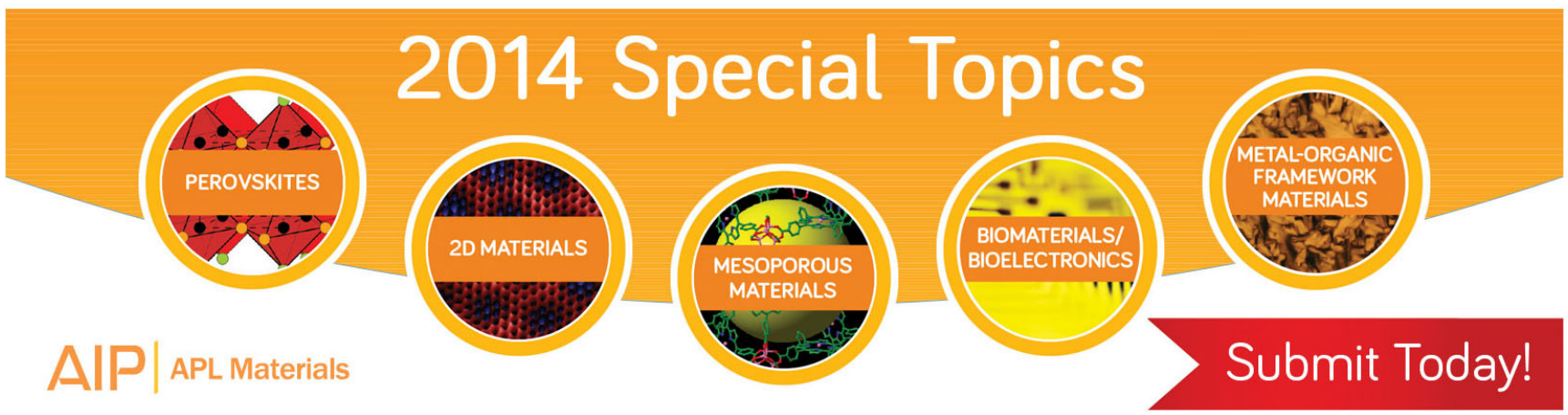




\title{
Pressure-dependent Schottky barrier at the metal-nanotube contact
}

\author{
Noejung Park ${ }^{\mathrm{a}, \mathrm{b})}$ \\ CSE Laboratory, Samsung Advanced Institute of Technology, P.O. Box 111, Suwon 440-600, Korea and \\ Department of Applied Physics, Dankook University, Seoul 140-714, Korea \\ Donghoon Kang \\ CSE Laboratory, Samsung Advanced Institute of Technology, P.O. Box 111, Suwon 440-600, Korea \\ Suklyun Hong \\ Department of Physics and Institute of Fundamental Physics, Sejong University, Seoul 143-747, Korea \\ Seungwu $\mathrm{Han}^{\mathrm{a}), \mathrm{c})}$ \\ Department of Physics and Division of Nano Science, Ewha Womans University, Seoul 120-750, Korea
}

(Received 25 January 2005; accepted 25 May 2005; published online 30 June 2005)

\begin{abstract}
We carry out first-principles density-functional calculations to investigate the electronic structure of the gold-carbon nanotube contact. It is found that a pressure applied on the gold-nanotube contact shifts the Fermi level from the valence edge to the conduction edge of the carbon nanotube. This can explain the $n$-type transport behavior frequently observed in the nanotube field-effect transistor using the gold as electrodes. An atomistic model is proposed for a possible origin of the pressure when the nanotube is embedded in the gold electrode. (C) 2005 American Institute of Physics.
\end{abstract}

[DOI: $10.1063 / 1.1990251]$

The carbon nanotube (CNT) is one of the promising materials for the next generation of electronic devices because of its intrinsic nanoscale size, one-dimensional geometry, and excellent transport properties. ${ }^{1,2}$ The high mobility, the large current density, and the controlled response to the gate voltage has been demonstrated in the nanotube-based fieldeffect transistor (FET). ${ }^{3-5}$ Significant research efforts are now brought to the large-scale integration of CNT-FETs, especially on issues such as isolation of individual nanotubes, sorting the metallic and semiconducting nanotubes, and patterning and integration of the electrodes and nanotubes. On the other hand, controlling the carrier type of CNT is a key issue for the development of a logic circuit, for example, nanotube-based complementary-metal-oxide semiconductor technology.

In earlier studies, it was widely accepted that the oxygen adsorption on the nanotube wall resulted in the $p$-type transport property of the FET made of as-grown nanotubes. ${ }^{6-8}$ However, the binding energy of the oxygen on the nanotube wall is rather weak and consequently the charge transfer is negligible. ${ }^{9-11}$ In addition, systematic experiments indicated that the oxygen molecules affect mainly the metal electrode rather than change the carrier density of the CNT body. ${ }^{12,13}$ It was shown that, irrespective of adhesion strength of the CNT to the metal electrode, the Fermi level pinning at the nanosized contact between metal and CNT is not strong. Thus, the Schottky barrier formed at the contact is determined mostly by the difference between the metal work function and the electronic affinity of the semiconducting nanotube. ${ }^{14-16}$ This raises a basic question as to why a gold electrode and CNTs without oxygen atoms frequently result in the FET having $n$-type transport behaviors. ${ }^{13}$ Since the gold surface has a work function $(\sim 5.3 \mathrm{eV})$ higher than that of the CNT $(\sim 4.8 \mathrm{eV})$, the Fermi level of the gold surface would be

\footnotetext{
a) Authors to whom correspondence should be addressed.

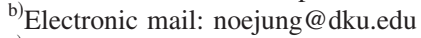

${ }^{c)}$ Electronic mail: hansw@ewha.ac.kr
}

closer to the valence edge and the FET device should show a $p$-type transport behavior. Motivated by this observation, we perform in this letter first-principles calculations on the metal-nanotube interface to investigate the Fermi level alignment at the contact in detail. It is found that the position of Fermi level of the metal electrode within the energy gap of semiconducting CNT is a function of the metal-nanotube distance and the external pressure present in the nanotubeelectrode interface can cause $n$-type behavior of electronic transport.

The density functional calculations are carried out using the computational code of the Vienna $a b$ initio simulation package. ${ }^{17}$ The plane-wave basis set with the energy cutoff of $400 \mathrm{eV}$ is employed to describe electronic wave functions. The ionic potentials are described by the projector augmented-wave pseudopotential ${ }^{18}$ and the exchange correlation energy of the electrons is approximated by the gradient-corrected potential suggested in Ref. 19. The atomic positions are relaxed until the Hellmann-Feynman force on each atom is reduced to within $0.05 \mathrm{eV} / \AA$.

For model geometries, we choose a semiconducting $(10,0)$ nanotube placed onto or in between the $\mathrm{Au}(111)$ surface. The model geometries and the corresponding projected density of states (PDOS) for the nanotube are shown in Fig. $1 .^{20}$ The surfaces are modeled as three gold layers. The unit cell contains 80 carbon atoms and 54 gold atoms and the periodic boundary condition is imposed by applying strain (corresponding to $2 \%$ compression) to the gold surface along the nanotube axis direction. Eight $k$-points are sampled in the irreducible Brillouin zone. In Fig. 1(a), only one side of the nanotube is in contact with the gold surface. The equilibrium distance $D$ of $3.01 \AA$ indicates that the adsorption is a physisorption type, and the contact resistance will be significant. This corresponds to a situation where a nanotube is freely placed on the metal electrode. Since gold has a larger work function, the Fermi level is aligned at the valence band edge of the carbon nanotube, as in Fig. 1(a).

In many experiments, however, the electrode is depos- 


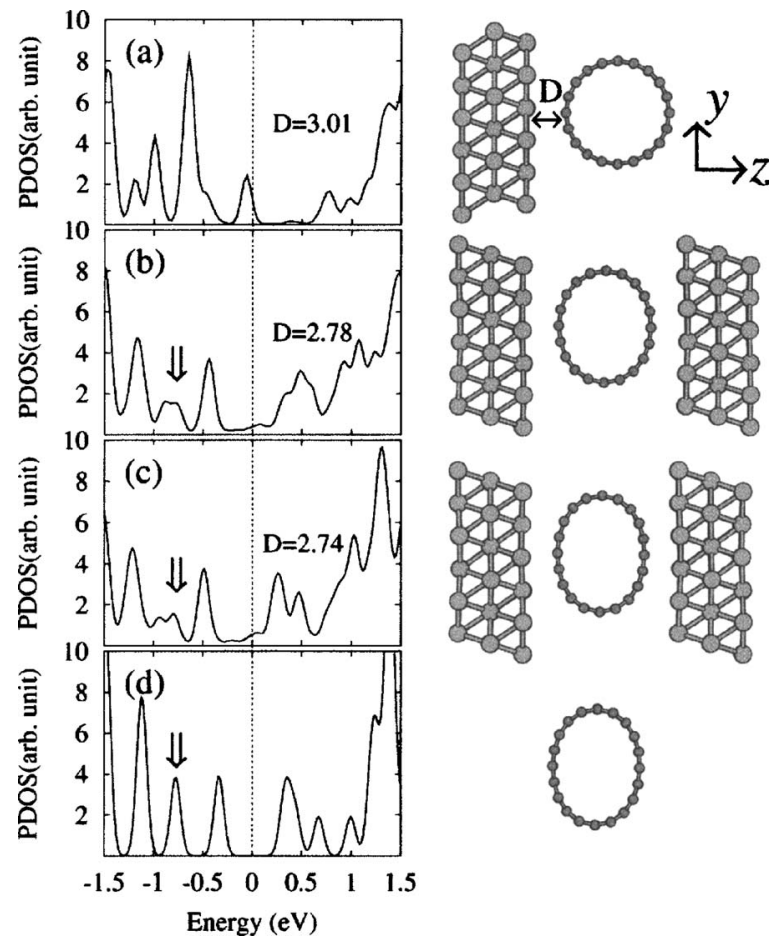

FIG. 1. The projected density of states for the $(10,0)$ carbon nanotube when it is (a) on the $\mathrm{Au}(111)$ surface; and (b) and (c) squeezed between gold slabs, respectively. The corresponding atomic structures are shown at the right of each density of state, with larger and smaller spheres representing gold and carbon atoms, respectively. The gold slab in the neighboring cell along the $z$ direction is drawn for convenience. (d) The density of states for the isolated carbon nanotube compressed as in (c).

ited lithographically after the nanotube is dispersed on the substrate. The kinetic energy of metal atoms during deposition and subsequent solidification will apply a finite pressure to the nanotube. ${ }^{21}$ To simulate the nanotube under this compressive force, we artificially press the metal-nanotube-metal contact. This is conveniently mimicked by reducing the unit cell length of the supercell along the $z$ direction [see Figs. 1(b) and 1(c)]. The most striking feature in Figs. 1(b) and 1 (c) is that as the contact is pressed, the Fermi level undergoes a relative upshift and thus become aligned closer to the conduction band of the nanotube. The CNT-FET including this configuration for the contact should show a $n$-type transport behavior. The calculated quantum-mechanical stress along the $z$ direction are 0.61 and 6.03 kbar for Figs. 1(b) and $1(\mathrm{c})$, respectively. The pressure-induced change of the transport behavior is consistent with the previous calculation reporting the increasing $p$-type barrier height as the metalnanotube distance decreases. ${ }^{22}$ The nonzero density of states in the band-gap region reflects the metal-induced gap states. Further increase of the compression will result in the metallization of the CNT. ${ }^{23}$

For comparison, the electronic density of states is plotted in Fig. 1(d) for the isolated $(10,0)$ nanotube with the atomic positions fixed at those in Fig. 1(c). We notice that the band structure is almost the same as that of the pristine nanotube. It is noticeable that the peaks corresponding to the second van Hove singularity are suppressed when the nanotube is compressed by the gold slabs, as indicated by arrows in Figs. 1(b) $-1(d)$. To understand this change in the PDOS, we examine the electronic distribution of three doubly degenerate valence band states at $\Gamma$ in Fig. 2 , as denoted by $V_{1}, V_{2}$, and $\mathrm{V}_{3}$. The phase change of the wave function indicated by \pm

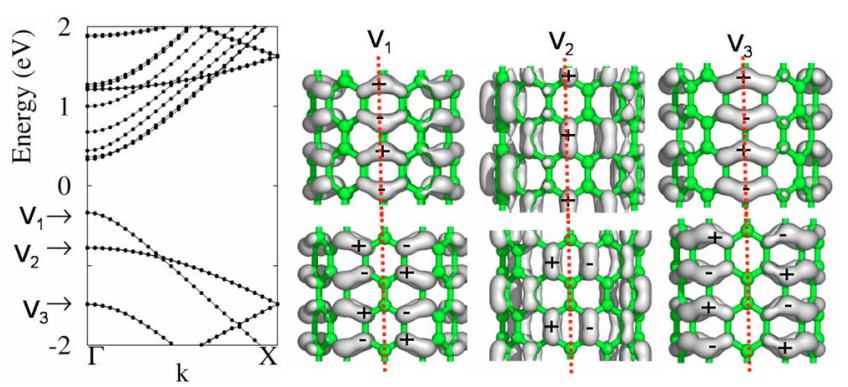

FIG. 2. (Color online) The band structure of the isolated, squeezed nanotube in Fig. 1(d). On the right, charge densities are shown for three doubly degenerate valence band states at $\Gamma$ point $\left(V_{1}, V_{2}\right.$, and $\left.V_{3}\right)$. The \pm indicates the sign of the wave functions. The dotted lines indicate the metal-nanotube contact line.

shows that the phase of $\mathrm{V}_{2}$ states changes most slowly along the nanotube-metal contact (dotted lines). Therefore, this state is easier to be hybridized with Au $6 s$ bands compared to the $V_{1}$ and $V_{3}$ states. This explains why the second van Hove singularity is strongly affected as the nanotube-metal distance is shortened. Consequently, the Fermi level upshift along with the suppression of the van Hove singularity shown in Figs. 1(b) and 1(c) is a consequence of increased occupation of Au $6 s$ orbitals through the hybridization with $\mathrm{V}_{2}$ states. (See the next paragraph.) The pressure-induced charge transfer from the nanotube to the gold surface will adjust the local work function of each system so that the band structure of the nanotube rigidly shifts down relative to that of the gold substrate.

In Figs. 3(a) and 3(b), the charge density differences for Figs. 1(a) and 1(c), $\Delta \rho=\rho(\mathrm{CNT} / \mathrm{Au})-\rho(\mathrm{CNT})-\rho(\mathrm{Au})$ are plotted along the $z$ direction after being averaged over the $x y$ plane. The origin of the $z$ axis corresponds to the position of the topmost gold layers and the arrows indicate the outermost carbon atoms. The electronic charge accumulates on the side of the gold surface, which indicates the larger electronegativity of gold and is consistent with the work function

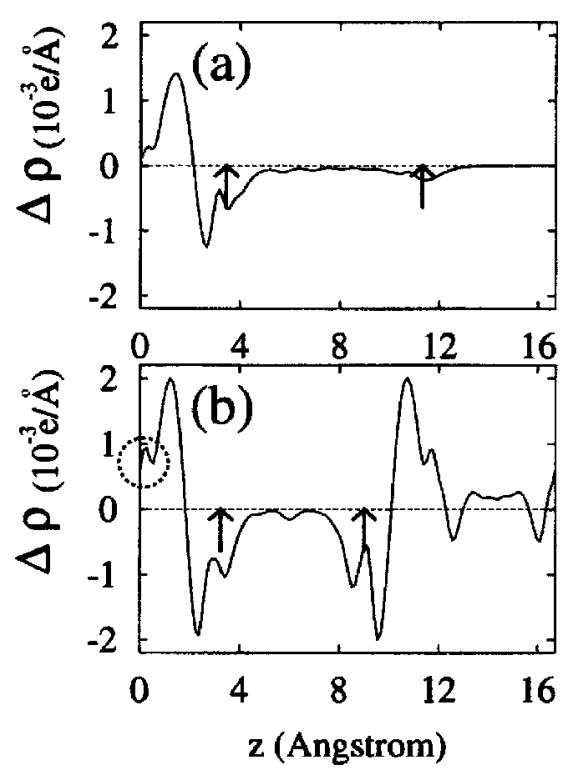

FIG. 3. The electronic charge densities for Figs. 1(a) and 1(c), obtained after subtracting those of isolated gold slabs and nanotubes, are plotted along the $z$ direction in (a) and (b) respectively. The abscissa is measured from the topmost gold layer. 


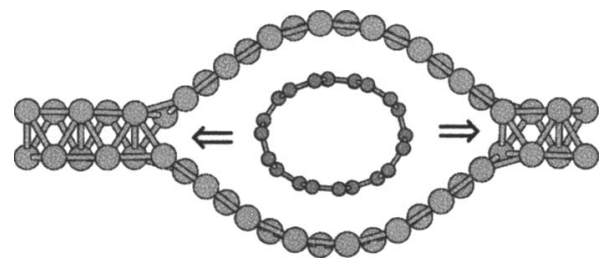

FIG. 4. Model geometry for the carbon nanotube embedded in the gold slab. The strain relaxation in the regions indicated by arrows results in the pressure on the carbon nanotube wall.

difference between CNT and gold. In Fig. 3(b), we note that more accumulation of the electron develops right above the gold surface (dashed circle) when the contact is under pressure. This comes from the specific $\mathrm{Au}-\mathrm{C}$ hybridization, as discussed in the previous paragraph. The charge transfer induced by the hybridization reduces the difference of local work functions between gold and CNT, pushing the Fermi level toward the conduction edge of CNT. We also perform a similar calculation for the $\mathrm{Au}(110)$ surface to check whether the charge transfer depends on the surface orientation. The result for the (110) surface is similar to that for the (111) surface; that is, the Fermi level moves from the valence edge to the conduction edge as the nanotube-metal distance is reduced.

Finally, we give a brief account on the origin of the pressure on the nanotube surface when the nanotube is embedded within the gold electrode. When evaporated gold particles are deposited on the dielectric substrate, a planar gold surface develops with the (111) orientation. Near the nanotube, the growth of the perfect (111) surface is inhibited and the broken bonds or the step edges are likely to be present nearby the nanotube wall. Even though detailed and systematic investigations on this subject is beyond the scope of the present work, a simplified model will be able to capture the essential features. In our model shown in Fig. 4, the $(5,5)$ nanotube is inserted between the two-layer gold slab. In the final relaxed structure obtained by density-functional calculations, the nanotube is found to be compressed by the gold layers. The strain present in the curved gold surface is balanced by the compression on the nanotube wall. This is analogous to the interface pressure when a small particle is embedded inside another material. ${ }^{24}$

One of the authors (S.H.) was supported by grants from the KOSEF through the Center for Nanotubes and Nanostructured Composites. Another (S.H.) was supported by the system IC 2010 program of the Korean government, by the National Program for 0.1 Terabit NVM Devices, and by the Sixth Strategic Supercomputing Support Program of KISTI.

${ }^{1}$ S. Iijima, Nature (London) 354, 56 (1991).

${ }^{2}$ S. Frank, P. Poncharal, Z. L. Wang, and W. A. de Heer, Science 280, 1744 (1998).

${ }^{3}$ S. J. Tans, A. R. M. Verschueren, and C. Dekker, Nature (London) 393, 49 (1998).

${ }^{4}$ A. Javey, H. Kim, M. Brink, Q. Wang, A. Ural, J. Guo, P. McIntyre, P. McEuen, M. Lundstrom, and H. Dai, Nat. Mater. 1, 241 (2002).

${ }_{5}^{5}$ A. Javey, J. Guo, Q. Wang, M. Lundstrom, and H. Dai, Nature (London) 424, 654 (2003).

${ }^{6}$ P. G. Collins, K. Bradley, M. Ishigami, and Z. Zettl, Science 287, 1801 (2000).

${ }^{7}$ G. U. Sumanasekera, C. K. W. Adu, S. Fang, and P. C. Eklund, Phys. Rev. Lett. 85, 1096 (2000).

${ }^{8}$ S.-H. Jhi, S. G. Louie, and M. L. Cohen, Phys. Rev. Lett. 85, 1710 (2000).

${ }^{9}$ H. Ulbricht, G. Moos, and T. Hertel, Phys. Rev. B 66, 075404 (2002).

${ }^{10}$ P. Giannozzi, R. Car, and G. Scoles, J. Chem. Phys. 118, 1003 (2003).

${ }^{11}$ S. Dag, O. Gülseren, T. Yildirim, and S. Ciraci, Phys. Rev. B 67, 165424 (2003).

${ }^{12}$ S. Heinze, J. Tersoff, R. Martel, V. Derycke, J. Appenzeller, and Ph. Avouris, Phys. Rev. Lett. 89, 106801 (2002).

${ }^{13}$ V. Derycke, R. Martel, J. Appenzeller, and Ph. Avouris, Appl. Phys. Lett. 80, 2773 (2002).

${ }^{14}$ F. Léonard and J. Tersoff, Phys. Rev. Lett. 84, 4693 (2000).

${ }^{15}$ Y. Xue and M. A. Ratner, Phys. Rev. B 69, 161402(R) (2004).

${ }^{16}$ Y. Yaish, J.-Y. Park, S. Rosenblatt, V. Sazonova, M. Brink, and P. L. McEuen, Phys. Rev. Lett. 92, 046401 (2004).

${ }^{17}$ G. Kresse and J. Hafner, Phys. Rev. B 47, 558 (1993); 49, 14251 (1994).

${ }^{18}$ P. E. Blöchl, Phys. Rev. B 50, 17953 (1994).

${ }^{19}$ J. P. Perdew, K. Burke, and M. Ernzerhof, Phys. Rev. Lett. 77, 3865 (1996).

${ }^{20}$ For calculating the PDOS, the Kohn-Sham eigenstates are projected onto the Wigner-Seitz spheres centered at each atom, whose radii are chosen to be 2 and $1 \AA$ for Au and C, respectively.

${ }^{21}$ B. Wei, P. K.-Redlich, U. Bäder, B. Heiland, R. Spolenak, E. Arzt, and M. Rühle, Ultramicroscopy 85, 93 (2000).

${ }^{22}$ B. Shan and K. Cho, Phys. Rev. B 70, 233405 (2004).

${ }^{23}$ S. Dag, O. Gülseren, S. Ciraci, and T. Yildirim, Appl. Phys. Lett. 83, 3180 (2003).

${ }^{24}$ D. A. Porter and K. E. Easterling, Phase Transformations in Metals and Alloys (Chapman \& Hall, 1992). 